\title{
ChemComm
}

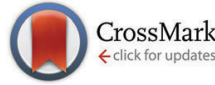

Cite this: Chem. Commun., 2014, 50, 12937

Received 9th July 2014

Accepted 26th August 2014

DOI: $10.1039 / c 4 c c 05301 j$

www.rsc.org/chemcomm

\section{Solid phase microextraction (SPME)-transmission mode (TM) pushes down detection limits in direct analysis in real time (DART) $\dagger$}

\author{
Germán Augusto Gómez-Ríos and Janusz Pawliszyn*
}

\begin{abstract}
A new SPME device was developed and applied for quick solventless extraction/enrichment of small molecules from complex matrices. Subsequently, the device was coupled as a transmission mode substrate to DART resulting in limits of detection in the low $\mathrm{pg} \mathrm{mL}^{-1}$ level in less than 3 minutes with reproducibility below $5 \%$ RSD.
\end{abstract}

In the last decade, ambient ionization methods have changed the way samples are analysed by mass spectrometry (MS). ${ }^{1}$ Several techniques, such as direct analysis in real-time (DART), desorption electrospray ionization (DESI), and paper spray (PS) have provided the scientific community with key tools for screening, pass/fail analysis, fingerprinting, and native sample imaging applications..$^{2-8}$ Today, most ambient MS approaches seek to record mass spectra without the need for sample preparation. ${ }^{1-4}$ Hence, the scientific community, usually trained on standard sample preparation/separation methods, may have numerous inquiries regarding ambient MS techniques, including their performance in terms of accurate and fast quantitative analysis. This includes inquiries over the suitability of such methods for trace analysis (e.g. low pg $\mathrm{mL}^{-1}$ ) in complex matrices, circumventing all sample preparation steps. With the use of such methods, analyses cannot always be performed in exceptionally short periods of time (i.e. $\leq 1$ minute). ${ }^{8-10}$ Generally, given that there is no sample pre-treatment, both the analysis time (i.e. time required to dry the sample onto the paper substrate ${ }^{10}$ and the linear dynamic range (i.e. diminished sensitivity by ion suppression) are likely to be sacrificed. ${ }^{11,12}$ As recently reviewed by Monge et al. and Venter et al. ${ }^{3,4}$ improvements in the experimental workflow are still needed in order to obtain better in situ analyte quantitation. Therefore, rather than subscribing to a no sample treatment technique, the use of minimal sample preparation could result in lower detection limits and more efficient analysis. To address the shortfalls described above, a solid phase microextraction (SPME) ${ }^{13-15}$ device that can be

Department of Chemistry, University of Waterloo, Waterloo, ON, Canada.

E-mail: janusz@uwaterloo.ca; Fax:+1-519-746-0435; Tel: +1-519-888-4567 ext.84641

$\dagger$ Electronic supplementary information (ESI) available: Detailed description of coated mesh devices, 12-mesh holder device, and calibrations on complex matrices. See DOI: 10.1039/c4cc05301j coupled as a transmission mode (TM) ${ }^{16-18}$ substrate to DART was developed. Succinctly, SPME-TM has been devised to act as an effective integration between sample preparation and ambient ionization. ${ }^{5}$ Essentially, the device consists of a stainless steel mesh $(74 \times$ $74 \mathrm{in}^{-1}$ wires, wire diameter 0.004 in) precisely coated on the strands with a biocompatible polymer $\left(\mathrm{C}_{18}\right.$-PAN $) \cdot{ }^{19} \mathrm{As}$ an SPME approach, the coated mesh $(\varnothing \leq 20 \mu \mathrm{m}$, Fig. 1) concurrently isolates and enriches the analytes of interest present in the sample matrix..$^{13}$ Additionally, given that undesirable interferences that might provide ionization suppression/enhancement are excluded from the sample during extraction (i.e. sample clean-up), detection limits are significantly enhanced. ${ }^{19,20}$ As a TM substrate, ${ }^{12}$ the coated mesh is positioned between the DART source and the mass spectrometer inlet (with all three coaxial to one another, $0^{\circ}$ angle); the stream of gas with metastable helium atoms flows through the mesh performing simultaneous desorption-ionization of the compounds sorbed on the surface of the coating particles. ${ }^{6}$ Subsequently, ions of the extracted/pre-concentrated analytes are transported into the atmospheric pressure interface (API) and analysed by tandem mass
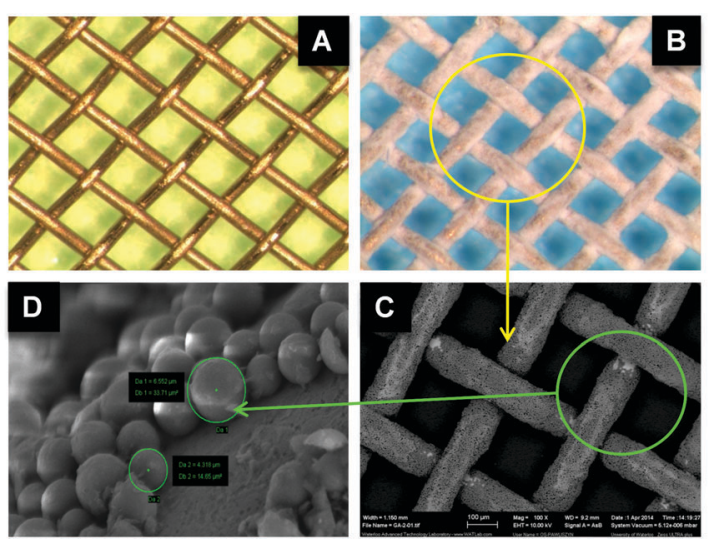

Fig. 1 SPME-TM coating characteristics: (A) microscope image of a bare SS mesh; (B) microscope image of a mesh coated with $C_{18}$ particles; (C) SEM image of the same mesh; (D) SEM image of layer coating particles on the mesh (particle size $\sim 5 \mu \mathrm{m}$ ). 


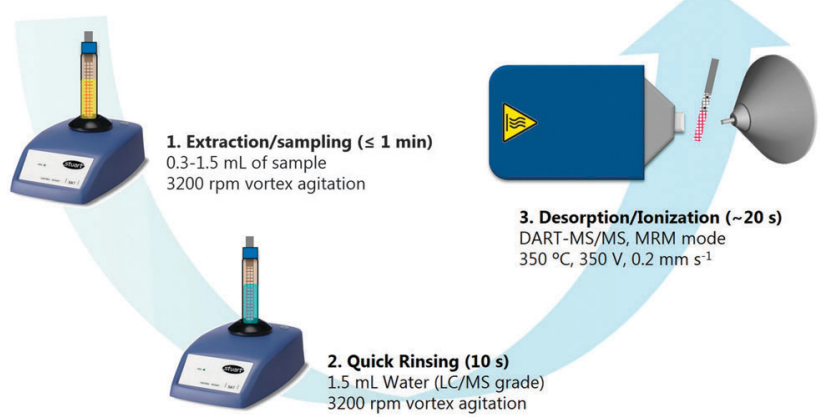

Scheme 1 Experimental set up for SPME-TM extraction from complex matrices and desorption-ionization using DART-MS/MS

spectrometry (MS/MS). ${ }^{21}$ As illustrated on Scheme 1, the analytical process by SPME-TM-DART-MS/MS consists of three simple steps.

First, a pre-conditioned coated mesh is immersed in a vial containing the sample matrix $(300-1500 \mu \mathrm{L})$ and fast enrichment is performed by agitating the sample at a high speed (e.g. vortex agitator; $t \leq 1 \mathrm{~min}$ ). Afterwards, the mesh is rapidly rinsed in a vial containing water $(t \leq 10 \mathrm{~s})$ to remove potential artefacts adhered to the coating surface. Lastly, the mesh is installed on a mesh-holder that allows for easy and fast replacement of up to twelve SPME-TM devices, and is adapted in an automatic linear rail that moves the mesh to the front of the DART source (Fig. S1, ESI $\dagger$ ).

To date, different geometries of SPME (i.e. fibre, in-tube, and thin-film) have been coupled to DART. ${ }^{20-23}$ However, most of them have evident drawbacks (Fig. S2, ESI $\dagger$ ). For example, as described by Cajka et al. ${ }^{22}$ the traditional SPME fibre requires cautious adjustment in front of the source to avoid severe fibre swinging and, consequently, irreproducible desorption-ionization of the analytes extracted. Although the early thin-film configuration reported by Mirnaghi et al. and Rodriguez-Lafuente et al. provided most SPME benefits, ${ }^{20,21}$ it was not an ideal TM substrate, since the coating, applied using brush painting, ${ }^{20}$ covered not only the strands but also the mesh openings. Although tiny random holes were placed on the coated mesh to allow the gas stream to flow through it, ion transmission was unfavourably affected (Fig. S2, ESI $\dagger$ ). ${ }^{21}$ Thus, the potential of the combination SPME-TM was not fully realized. Recently, Wang et al. disclosed the first on-line coupling of the in-tube (IT) configuration to DART. $^{23}$ In this work, the authors demonstrated that IT-SPME is a sensitive method for the determination of trace pesticides in juice/ water (Limit of quantitation (LOQ) $\sim 0.2 \mathrm{ng} \mathrm{mL}{ }^{-1}$ ). However, the operation of the system is cumbersome and requires extra instrumentation (i.e. syringe pump to control solvent desorption flow). In addition, prior to the IT-SPME sampling, the sample requires centrifugation and filtration; otherwise, the extraction material might get clogged with particulates, fibres, or proteins from the matrix. ${ }^{23}$ In contrast to the applications listed above, the present contribution describes multiple SPME-TM devices that can be easily and steadily installed on a 12-position holder and accurately positioned between the DART source and the mass spectrometry inlet (Fig. S1, ESI ${ }^{\dagger}$ ). In addition, given that the mesh is exclusively coated on the strands, efficient desorption-ionization and ion transmission is obtained. Finally, unlike IT-SPME, SPME-TM requires neither additional apparatus nor sample pre-treatment. Although the method herein described is not the first coupling of SPME to DART-MS, it is undeniably the most comprehensive and simple approach proposed to date. ${ }^{5}$

In the past, it has been incorrectly assumed by scientists not familiar with SPME that extraction/enrichment cannot be performed in short periods of time, ${ }^{1-3}$ and that extractions exclusively performed at equilibrium can achieve low LOD/LOQ. ${ }^{14,24}$ However, contrary to general assumptions, direct coupling of SPME to mass spectrometry easily outperforms traditional detection limits with remarkably brief extraction times due to several reasons. First, the dilution factor inherent in most SPME-LC methods is removed from the analytical procedure. ${ }^{25}$ Second, the high surface area contact between the extraction phase and matrix facilitates high mass transfer rates, while thin coatings ensure rapid equilibration times and efficient desorption to the mass spectrometer. Hence, the LOD is mainly constrained by the detection capabilities of the MS system rather than by the built-in features of the coating. ${ }^{15}$ Preliminary experiments in our laboratory using thin-film microextraction devices (TFME, blade geometry ${ }^{24}$ ) showed that 15 seconds is sufficient to extract a quantifiable amount of analyte at the low ppb level even when using the traditional LC/MS approach (Fig. S3, ESI $\dagger$ ). Indeed, if lower LODs are required, the interaction time between the coating and the sample matrix can be increased. ${ }^{14}$ For instance, LOQs as low as 2 and $19 \mathrm{pg} \mathrm{mL}^{-1}$ were reached when performing 1 minute extractions from $1.5 \mathrm{~mL}$ of phosphate buffered saline (PBS) spiked with cocaine and diazepam (DZP), respectively. Furthermore, the linear dynamic range of the method, evaluated from $10 \mathrm{pg} \mathrm{mL}^{-1}$ up to $50 \mathrm{ng} \mathrm{mL}{ }^{-1}$, showed astounding linearity (Fig. S4, ESI $\dagger$ ). It is worth emphasizing that higher concentration levels are not a limitation for SPME; ${ }^{14}$ thus, the scope of this report is to introduce the remarkable quantitation capabilities at trace levels of the SPME-TM-DART-MS/ MS, rather than study the indisputable. However, in cases where a compound is present at a high concentration (i.e. ppm levels) and the affinity of the coating for the analyte is strong, even shorter extraction times (e.g. $\leq 30 \mathrm{~s})$ can be performed.

A noteworthy feature SPME-TM devices in comparison with other ambient mass spectrometry apparatus is their reproducibility and potential for reusability. ${ }^{20}$ Extractions performed with 9 independent SPME-TM devices $(n=36)$ from $1.5 \mathrm{~mL}$ of PBS solution spiked with cocaine and diazepam showed intra-/inter-device reproducibility lower than 4.7 and 3.2\%, respectively (Table 1; Tables S1 and S2, ESI $\dagger$ ). In addition, the extraction phase is able to withstand well the extractiondesorption cycles. However, although a decrease on the peak area signal use for quantitation after four consecutive uses was not observed, further experiments are required to determine the longterm durability of the SPME-TM devices. Certainly, it has been confirmed that by using thin-coatings, efficient mass transfer of analytes (fast extractions) and effective desorptions can be achieved. ${ }^{13}$ Furthermore, while the signals obtained on a second desorptionionization cycle (carry-over) were approximately $5 \%$ of the signal used for quantitation of DZP (Fig. S5, ESI $\dagger$ ), it is important to highlight that detection of DZP and cocaine was performed concomitantly. Thus, DART experimental conditions were not exclusively optimized for DZP, which could explain why a small fraction of the analytes still remained after the first desorption-ionization cycle. ${ }^{26}$ Nevertheless, by implementing a cleaning step shortly after the desorption-ionization 
Table 1 SPME-TM inter-device reproducibility. SD, standard deviation; RSD, relative standard deviation. Ratio results correspond to the average of extractions performed with 9 independent devices $(n=36)$ from a PBS solution spiked with $20 \mathrm{ppb}$ of each analyte

\begin{tabular}{|c|c|c|c|c|c|}
\hline Experiment & $\begin{array}{l}\text { Ratio } \\
{[\mathrm{A} / \mathrm{Is}]}\end{array}$ & SD & $\begin{array}{l}\text { RSD } \\
{[\%]}\end{array}$ & $\begin{array}{l}\text { Carryover DART } \\
{\left[\mathrm{A}_{\mathrm{c}} / \mathrm{A}_{\mathrm{i}}\right][\%]}\end{array}$ & $\begin{array}{l}\text { Carryover solvent } \\
{\left[\mathrm{A}_{\mathrm{c}} / \mathrm{A}_{\mathrm{i}}\right][\%]}\end{array}$ \\
\hline Diazepam & 1.8 & 0.05 & 3 & 5 & 0.3 \\
\hline Cocaine & 1.6 & 0.05 & 3 & 2.4 & 0.2 \\
\hline
\end{tabular}

cycle (i.e. mixture of methanol, isopropanol and acetonitrile; $50: 25: 25)$ negligible carry-over was attained $(\leq 0.4 \%)$. Undeniably, the cleaning step should be optimized according to both the chemistry of the coating and its affinity towards the analyte of interest. ${ }^{14}$ In cases where there is an extensive variation in analyte concentration among samples (i.e. low ppt to ppm levels), SPME-TM devices should be restricted to a single use. Otherwise, a small portion of the analytes could remain on the coating, even after the cleaning cycle, which could lead to potential false positives. ${ }^{25}$ A possible solution while working with compounds at concentrations greater than $50 \mathrm{ppb}$ and with high affinity towards the coating would be to perform shorter extractions $(\leq 30 \mathrm{~s})$. Thus, the amount of analyte enriched would be diminished and the exhaustive removal of analytes non-desorbed by DART would be plausible with a cleaning step.

Despite the advantages of paper spray (PS) for the analysis of small volumes of untreated samples, Li et al. and Espy et al. reported that the use of paper substrate requires a drying step, either with air or electromechanical assistance, prior to desorption-ionization, which extends the total time of analysis. ${ }^{9,27}$ In contrast to PS, analytes extracted by SPME-TM can be desorbed immediately after a quick rinse in water $(t \leq 10 \mathrm{~s})$ and gentle removal of excess water with a cleaning tissue $(t \leq 2 \mathrm{~s})$. An exceptional characteristic of SPME-TM is the mechanical strength provided by the mesh-blade arrangement used to build its structure (Fig. S6, ESI†). As a result, deformation/ damage of the device hardly occurs regardless of the sample dimensions or its characteristics. Thus, SPME-TM could be used to perform extractions from limited sample volumes (e.g. extractive blood spot ${ }^{20}$ ) up to large volumes (e.g. on-site monitoring of a creek/river ${ }^{13}$ ).

MS analysis provides significant amounts of information about complex samples. ${ }^{1}$ However, sample pre-treatment required before MS analysis not only is labour-intensive and time-consuming, but also intricate. ${ }^{3,4}$ Contrastingly, due to its speed and ease of use, SPME-TM is an ideal device for the screening of controlled substances in biological samples as well as for therapeutic drug monitoring (TDM). ${ }^{20,21,25}$ To demonstrate its applicability, SPME-TM was used for the quantification of cocaine and DZP in urine and plasma. Fig. 2 (Fig. S7, ESI $\dagger$ ) summarizes the exceptional linearity achieved in both matrices. Similar to PBS, LOQs of 2 and $5 \mathrm{pg} \mathrm{mL}^{-1}$ were determined for cocaine in urine and plasma, respectively (Table S3, ESI $\dagger$ presents comparison versus LOD/LOQ by LC/MS). Thus, matrix effects are significantly minimized by the sample clean-up provided by SPMETM, and analytes with low binding present comparable detection limits independently of the matrix. ${ }^{14}$ By removing salts and biomolecules that mechanically attach to the coated strands during the extraction, the rinsing step extends the operative time of the mass spectrometer, providing reliably high instrumental sensitivity as well as minimizing instrument maintenance. ${ }^{21}$ Unlike cocaine, the LOQ

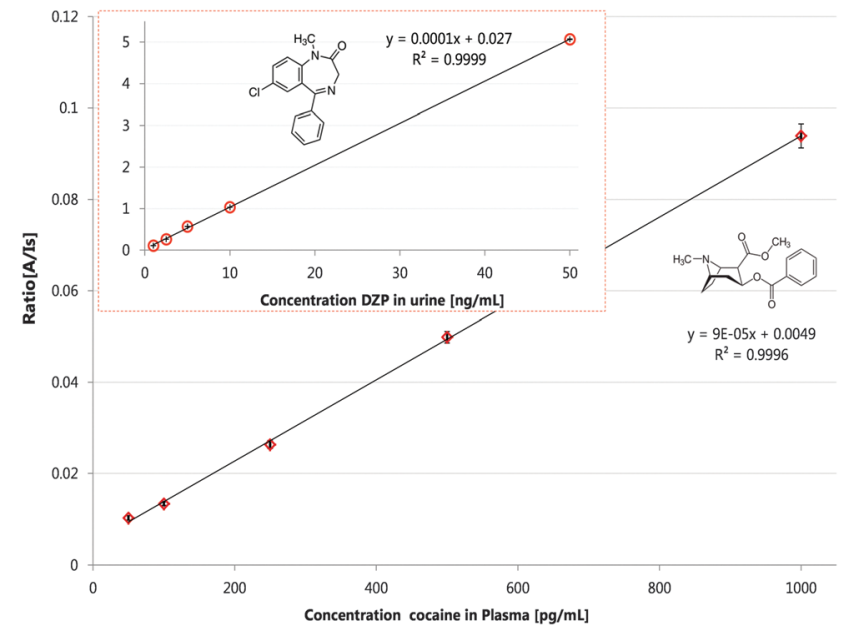

Fig. 2 Quantitative analysis of urine spiked with cocaine $\left(50 \mathrm{pg} \mathrm{mL}^{-1}\right.$ to $\left.1 \mathrm{ng} \mathrm{mL}^{-1}\right)$ and its isotopologue $\left[\mathrm{D}_{3}\right]$ cocaine $\left(12 \mathrm{ng} \mathrm{mL}^{-1}\right)$. Insert plot shows quantitative analysis of plasma spiked with DZP $\left(1 \mathrm{ng} \mathrm{mL}^{-1}\right.$ to $\left.50 \mathrm{ng} \mathrm{mL}^{-1}\right)$ and its isotopologue $\left[D_{5}\right]$ diazepam $\left(12 \mathrm{ng} \mathrm{mL}^{-1}\right)$. Bars represent the standard deviation of analysis for three replicates with independent SPME-TM devices.

for DZP in plasma (497 pg $\mathrm{mL}^{-1}$ ) was significantly higher in comparison to urine and PBS (19 and $28 \mathrm{pg} \mathrm{mL}^{-1}$, respectively). However, it is worth mentioning that DZP is $98 \%$ bound to plasma proteins and, as an SPME-based approach, the TM configuration only extracts the free-portion of analytes in the sample. ${ }^{19}$ In addition, since the TM configuration guarantees homogeneous interaction between extracted and ionizing species, standard-free quantitation is also feasible with SPME-TM. Nevertheless, given that extraction is not performed at equilibrium ( $t \leq 1 \mathrm{~min}$ ), precise variables should be cleverly controlled in order to obtain reproducible and repeatable results, namely sampling time, convection, as well as coating thickness homogeneity. ${ }^{19}$ Definitely, devices having consistent coating distribution, composition, and thickness are needed to ensure reproducible extraction of the analytes to the coating and desorption of the analytes from the coating. Different to other direct ionization techniques, given that no sample matrix is placed in front of the mass spectrometer, homogeneous coatings are required to normalize the sample matrix by always extracting the same amount of analyte of interest independently of the device used. Hence, reproducible coatings not only minimize sample interferences, but also provide reproducible instrumental response and no internal standard is required to achieve reproducibility below 15\% RSD (Fig. S8, ESI $\dagger$ ).

As a proof-of-concept, SPME-TM was used to simultaneously monitor 21 prohibited substances spiked on PBS at $20 \mathrm{ng} \mathrm{mL} \cdot$. $^{-1,28}$ Selected reaction monitoring (SRM) was used to exclusively identify each compound. LOD were tentatively predicted based on the results obtained for cocaine and DZP in PBS. Even though DART source parameters were not optimized for each analyte, all substances were detected, and 16 compounds provided hypothetical detection limits lower than $50 \mathrm{pg} \mathrm{mL}^{-1}$ (e.g. heroin [ $\log P$ 1.52], propranolol [ $\log P$ 3.48], and stanozolol [ $\log P$ 5.53]; Fig. S9, Table S4, ESI $\dagger$ ). Insofar as SPME-TM derives its sensitivity and selectivity from the physicochemical properties of the extraction phase, current research is focused on the development of new devices with greater affinity towards specific target compounds. Certainly, the ability of SPME-TM to screen numerous 
substances in a single analysis, without forfeiting sensitivity or quickness, is a noteworthy characteristic of this technique, which could be used in other applications such as monitoring of personal care products in waste water or pesticides in food commodities. ${ }^{13}$

An asset of the mesh-blade arrangement of SPME-TM is that it can be used to perform either individual extractions (i.e. from a vial containing sample) or high-throughput extractions in a multi-well plate format (Fig. S10, ESI $\dagger$ ). Similar to other SPME geometries, SPME-TM can be adapted to concurrently analyse up to 96 samples in a single run. ${ }^{19,24}$ Hence, by automating the extraction/rinsing step, as well as the desorption step with the aforementioned system, a total analysis time of 60 seconds or less could be attained per sample. Undoubtedly, the multiple benefits of SPME-TM, such as low detection limits and minimal matrix interferences, should stimulate the scientific community to use a swift sample preparation approach prior to direct introduction to MS analysis.

In summary, a novel SPME device that can be easily coupled to DART for targeted and quantitative trace analysis (ppt to ppm levels) was developed. Given the structural configuration of the apparatus, it can be used to perform extractions independently of the sample complexity and its dimensions. Contrary to popular belief, ${ }^{1-5}$ analyte-enrichment and sample-clean-up with SPME-TM can be performed in less than 1 minute, with total analysis time not exceeding 3 minutes. In addition, since non-solvent is required in the entire process, and the device can be used on-site, the whole analytical process with SPME-TM is "green". Unquestionably, better understanding of the fundamentals driving the extraction, as well as ion transportation would lead to lower the detection limits further currently attained by SPME-TM. ${ }^{26}$ Continuous improvement in design of instrument sensitivity will aid this goal. Moreover, sensitivity can be enhanced by precisely tuning features of both techniques such as: (a) the substrate characteristics (i.e. mesh material type, empty space diameter, consecutive hole to hole distance, and strand size); ${ }^{29}$ (b) the coating features (i.e. polymeric phase chemistry, particle size, porosity, thermal conductivity, thermal stability, and affinity for the analyte of interest), ${ }^{15}$ and (c) the position of the substrate in which there is a balance between efficient neutral generation by thermal desorption and transport into the mass spectrometer (i.e. DART source operative conditions such as: gas temperature and flow, discharge voltage, grid electrode voltage, spatial position of the mesh in relation to the ion source nozzle). ${ }^{17,26}$ A comprehensive optimization of the variables described above will certainly boost the performance of SPMETM technique herein discussed.

Although SPME-TM reusability is advantageous for highthroughput applications in which a hefty amount of samples should be processed daily, ${ }^{19}$ it is also envisaged as a disposable device for in situ trace analysis. ${ }^{13}$ By coupling SPME-TM to deployable MS/MS systems, truly "real-time" and quantitative analysis of complex mixtures will be delivered on-site. ${ }^{9,30}$ Therefore, due to the unique combination of speed, selectivity, sensitivity, reproducibility and simplicity, SPME-TM-DARTMS/MS is a suitable rapid screening and quantitation technique not only for point-of-care TDM, but also in numerous environmental, food and forensic applications. ${ }^{31}$
The authors thank the Natural Sciences and Engineering Research Council (NSERC) of Canada, Supelco, IonSense Inc., and VBM Science Ltd. for the financial support. We also thank the University of Waterloo Science Shop the technical support, as well as Nathaly Reyes-Garcés, Barbara Bojko, and Kaveh Amini for valuable scientific discussions.

\section{Notes and references}

1 R. G. Cooks, Z. Ouyang, Z. Takats and J. M. Wiseman, Science, 2006, 311, 1566-1570.

2 R. B. Cody, J. A. Laramée and H. D. Durst, Anal. Chem., 2005, 77, 2297-2302.

3 M. E. Monge, G. A. Harris, P. Dwivedi and F. M. Fernandez, Chem. Rev., 2013, 113, 2269-2308.

4 A. R. Venter, K. A. Douglass, J. T. Shelley, G. Hasman Jr and E. Honarvar, Anal. Chem., 2014, 86, 233-249.

5 J. Deng, Y. Yang, X. Wang and T. Luan, TrAC, Trends Anal. Chem., 2014, 55, 55-67.

6 J. H. Gross, Anal. Bioanal. Chem., 2014, 406, 63-80.

7 K. J. Ewing, D. Gibson, J. Sanghera and F. Miklos, Anal. Chem., 2013, 85, 9508-9513.

8 J. Liu, H. Wang, N. E. Manicke, J. Lin, R. G. Cooks and Z. Ouyang, Anal. Chem., 2010, 82, 2463-2471.

9 L. Li, T. Chen, Y. Ren, P. I. Hendricks, R. G. Cooks and Z. Ouyang, Anal. Chem., 2014, 86, 2909-2916.

10 H. Wang, J. Liu, R. Graham Cooks and Z. Ouyang, Angew. Chem., Int. Ed., 2010, 49, 877-880.

11 E. Crawford, J. Gordon, J. Wu, B. Musselman, R. Liu and S. Yu, Bioanalysis, 2011, 3, 1217-1226.

12 C. M. Jones and F. M. Fernandez, Rapid Commun. Mass Spectrom., 2013, 27, 1311-1318.

13 B. Bojko, E. Cudjoe, G. A. Gómez-Ríos, K. Gorynski, R. Jiang, N. ReyesGarcés, S. Risticevic, E. A. S. Silva, O. Togunde, D. Vuckovic and J. Pawliszyn, Anal. Chim. Acta, 2012, 750, 132-151.

14 S. Risticevic, H. Lord, T. Górecki, C. L. Arthur and J. Pawliszyn, Nat. Protoc., 2010, 5, 122-139.

15 E. A. Souza Silva, S. Risticevic and J. Pawliszyn, TrAC, Trends Anal. Chem., 2013, 43, 24-36.

16 J. E. Chipuk and J. S. Brodbelt, J. Am. Soc. Mass Spectrom., 2008, 19, 1612-1620.

17 J. J. Pérez, G. A. Harris, J. E. Chipuk, J. S. Brodbelt, M. D. Green, C. Y. Hampton and F. M. Fernández, Analyst, 2010, 135, 712-719.

18 G. A. Harris, C. E. Falcone and F. M. Fernandez, J. Am. Soc. Mass Spectrom., 2012, 23, 153-161.

19 D. Vuckovic, E. Cudjoe, F. M. Musteata and J. Pawliszyn, Nat. Protoc., 2010, 5, 140-161.

20 F. S. Mirnaghi and J. Pawliszyn, Anal. Chem., 2012, 84, 8301-8309.

21 A. Rodriguez-Lafuente, F. S. Mirnaghi and J. Pawliszyn, Anal. Bioanal. Chem., 2013, 405, 9723-9727.

22 T. Cajka, K. Riddellova, M. Tomaniova and J. Hajslova, J. Chromatogr. A, 2010, 1217, 4195-4203.

23 X. Wang, X. Li, Z. Li, Y. Zhang, Y. Bai and H. Liu, Anal. Chem., 2014, 86, 4739-4747.

24 D. Vuckovic, TrAC, Trends Anal. Chem., 2013, 45, 136-153.

25 E. Boyaci, K. Gorynski, A. Rodriguez-Lafuente, B. Bojko and J. Pawliszyn, Anal. Chim. Acta, 2014, 809, 69-81.

26 G. A. Harris and F. M. Fernandez, Anal. Chem., 2009, 81, 322-329.

27 R. Espy, S. A. Teunissen, N. E. Manicke, Y. Ren, Z. Ouyang, A. van Asten and R. G. Cooks, Anal. Chem., 2014, 86, 7712-7718.

28 A. Thomas, H. Geyer, W. Schänzer, C. Crone, M. Kellmann, T. Moehring and M. Thevis, Anal. Bioanal. Chem., 2012, 403, 1279-1289.

29 J. E. Chipuk and J. S. Brodbelt, J. Am. Soc. Mass Spectrom., 2009, 20, 584-592.

30 J. M. Wells, M. J. Roth, A. D. Keil, J. W. Grossenbacher, D. R. Justes, G. E. Patterson and D. J. Barket Jr, J. Am. Soc. Mass Spectrom., 2008, 19, 1419-1424.

31 B. Bojko, K. Gorynski, G. A. Gomez-Rios, J. M. Knaak, T. MacHuca, E. Cudjoe, V. N. Spetzler, M. Hsin, M. Cypel, M. Selzner, M. Liu, S. Keshjavee and J. Pawliszyn, Lab. Invest., 2014, 94, 586-594. 